\title{
PACKET OPTIMIZATION IN ADAPTIVE COGNITIVE RADIO SENSOR NETWORKS USING OFDM
}

\author{
Ravishankar Kandasamy, Assistant Professor, Paavai Engineering College \\ Dr.M.Sudha, Professor and Head, Paavai Engineering College \\ P.Durga, Final Year, ECE, Paavai Engineering College \\ R.Devishree, Final Year, ECE, Paavai Engineering College \\ R.Abinaya, Final Year, ECE, Paavai Engineering College
}

\begin{abstract}
:
With the rapid development of digital communication, the demand for spectral resources is increasing and the building of cognitive radios is the right solution for that. Cognitive radio networks are designed to utilize the licensed spectrum when it is not used by the primary licensed users. We are going to propose a method for effective data transmission and streaming in cognitive radio networks. By that we can achieve energy efficiency, less power consumption and much more transmitted information. In this paper, we are going to employ the OFDM method of spectrum sensing and proposed to use Cognitive Radio MAC protocol. Further our main technique is to divide the data packets into different sizes for transmission. The simulation results reveal that there is a better improvement in the detection of idle channel in the cognitive radio network and the delay is reduced with high quality transmission.
\end{abstract}

\section{INTRODUCTION}

With huge development in communication applications, the spectrum becomes more congested and the need for data rate is also highly increased. Radio spectrum is a limited resource and the service is allocated by fixed spectrum alignment[1].So, some frequencies are heavily used and some other band frequencies are weakly used.The number of devices or users using the unlicensed

Copyright (C) Author spectrum is higher which results in spectrum scarcity

.To overcome the spectrum demand issue, cognitive radio networks are designed to utilize the licensed spectrum whenit is not used by licensed users. CR is a promising technique to improve spectral utilization. Spectrum is allocated dynamically in the CR networks which further increases the spectrum utilization. In spectrum sensing mechanism, we are using OFDM method of spectrum sensing.

Here, the unlicensed users are going to use the spectrum of the primary users which is not in use. It senses the spectrum and finds the vacant spectrum available in the network[3,5]. Then, it chooses the best spectrum present which meets the required quality of service by the secondary user. The unlicensed users leave the spectrum as soon as the licensed user returns.

The ability to improve the spectral efficiency, enhance network efficiency and serve the telecommunication, user with high quality of user services is performed by the application of ODFM spectrum sensing[9]. The capabilities of CR allow optimization of data packets into various sizes and the resultant parameters shows that transmission frequency, waveform, interference and bandwidth performance are better. 


\section{PROBLEM DEFINITION AND SOLUTION}

Due to improper packet size, data transmission in wireless networks is quite complicated This is the problem we are facing in wireless communication[8]. This can be rectified by dividing the data packet into appropriate sizes. As we are going to do the channel allocation for streaming in the cognitive radio networks, We took many references for a survey of cooperative spectrum sensing to know the rapid growth of the wireless communication and its demand. We observed the classification and framework of the cooperative sensing to improve the efficiency of the system. There are three ways in the cooperative sensing classification. They are centralized, distributed and the relay assisted.[1] The knowledge base of the cognitive sensing includes the radio environment map, channel gain map, power spectral density map, received signal strength profiles, primary user activity and statistics and primary user localization $\&$ tracking. In cognitive radio, the sensing time is proportional to the number of samples that we take. So, an efficient model can be created by this proportionality[8]. The energy efficiency of the cognitive radio can be improved by the threshold interferences at the primary and secondary level. The calculations of the sound to noise ratio to the peak power has provide a lot of information about the energy efficiency. The energy efficiency and spectrum utilization can be increased by increasing the secondary users than the available channels.

The performance of cognitive spectrum sensing based on the OFDM with optimized packet size helps in better throughput, inter operability, less interference and high energy efficiency.The transmitted signals are received with multiple reflections, shows that medium size packets travels with less delay[5].
Published Online 20-March-2021

This saves the time efficiently in different high definition video resolutions with different frame rates. The radio spectrum management is a limited source controlled by regulations and the recognized authorities[5]. They allocate radio spectrum by assigning the channels to specific users with licenses for use the specific wireless technologies. There are different metrics used in this for the performance evaluation of the cognitive system.

The software defined radio avoids the frequent modification in the hardware structure with the use of software defined protocols. This implementation of OFDM based spectrum sensing method in cognitive radio is an effective way for real time transmission[1]. This can be extended for simultaneous transmission of multiple signals and use of multiple frequency band.

\section{RELATED WORKS:}

In adaptive cognitive radio sensor network few papers describes that packet sizes will result in effective transmission but there is no clear results. Several papers using MAC protocols in CRSN networks and modeled the concepts results in inter symbol interference and collisions[7]. As far as the data packets are concerned the migration from high definition to ultra-high definition is already underway. Cognitive radio networks provide high bandwidth to mobile users via heterogeneous wireless architecture and dynamic spectrum access techniques. Basically, it avoids the congestion by changing the unused radio spectrum that is not noted down by engaged through a licensed user. This is the most promising and efficient technique for the bandwidth utilization of each and every band. Threshold level of the OFDM based spectrum detection depends on the noise variance. It cannot differentiate between the interference from the other secondary users sharing the same channel and with the primary user. Its 
performance degrades under low signal to noise ratio or with noise uncertainty[6].

Analytical models based on this concept are more complexity and imperfect inthroughput and end nodes. Moreover there is no major research based on varying the sizes of the packet for effective data transmission.

\section{VIDEO STREAMING SYSTEM MODEL}

For application in the multimedia field it demands more bandwidth for the transmission. For video it needs more storage and bandwidth in uploading and downloading. Therefore, the image is divided into $n^{*} \mathrm{~m}$ blocks and every block $\mathrm{p}=\mathrm{nm}$ had a pixel value of $A=\left\{A_{1}, A_{2}, A_{3} \ldots A p\right\}$

Then, we can calculate the first and second samples and their variance by using the equations,

$$
\begin{gathered}
\bar{A}=\frac{1}{p} \sum_{i=1}^{p} A_{i} \\
\overline{A^{2}}=\frac{1}{p} \sum_{i=1}^{p} A_{i}^{2} \\
\text { Variance } \sigma^{2}=\overline{A^{2}}-\overline{(A)^{2}}
\end{gathered}
$$

For a design of only one-bit quantized value, we set the threshold value as $A_{t h}$ and the output levels can be encoded as $a, b$.

Let $\mathrm{L}=\mathrm{A}_{\mathrm{i}}$ 's greater than the threshold value $\left(A_{t h}\right)$.

$$
\begin{aligned}
& \text { If } \mathrm{A}_{\mathrm{i}}>\mathrm{A}_{\text {th }} \text {,output is } \mathrm{b}=\bar{A}+\sigma \sqrt{\frac{p-L}{L}} \\
& \text { Otherwise, output is } \mathrm{a}=\bar{A}-\sigma \sqrt{\frac{p-L}{L}}
\end{aligned}
$$

Sensing the alteration in the data may affect the estimation of global decision for access the licensed spectrum of cognitive radio. So, we assume the presence of $\mathrm{j}$ number of secondary users, malicious users, and normal users. Secondary users sensing the primary by using the received signal $\mathrm{x}(\mathrm{t})=\left\{\mathrm{x}_{1}(\mathrm{t}), \mathrm{x}_{2}(\mathrm{t}) \ldots, \mathrm{xj}(\mathrm{t})\right\}$. According to the binary sensing report the jth secondary user can be given as

$$
\mathrm{L}_{\mathrm{j}}(\mathrm{K})= \begin{cases}1 & x(t)>\lambda, \\ 0 & x(t)<\lambda\end{cases}
$$

Where $\lambda$ is the threshold value and $\mathrm{K}$ represents the time interval.

Secondary users senses and sends one bit of local sensing report to the fusion center that takes a global decision based on the rules. The decision $\mathrm{O}(\mathrm{K})$ is given as,

$$
\mathrm{O}(\mathrm{K})=\left\{\begin{array}{l}
1, \sum_{1}^{j} L(j) \geq M \\
0, \Sigma_{1}^{j} L(j)<M
\end{array}\right.
$$

\section{Spectrum Sensing from Licensed Users}

In a cognitive radio network, secondary unlicensed users are opportunistically allowed to utilize licensed spectrum band when the primary user or licensed user is not present in the cognitive radio environment. Energy is consumed by these secondary users in utilizing the licensed spectrum band before giving up access to the primary user. Here, we give access to SU's to consume the primary users network by spectrum sensing in a cognitive radio network[5].

The secondary users detect the free channel present in the transmission network. By means of sensing the primary user's regular usage pattern, we can predict the number of channels which is freely available without involving any kind of transmission and reception during a certain period of time. The energy consumption, network usage rate and the regular working pattern in each region of the primary user's activity is analyzed and presented. Thus, it will be easy for the unlicensed users to utilize the free region when they are not in use.

\section{Sensing and Hypothesis Model}

The sensing is done by OFDM technique by considering the radio channel 
parameters such as transmission channel characteristics, interference level, noise level, spectrum availability, power availability, etc... which is done in frequency and time domain[11]. It can also be done in code and phase domains as well. The system consists of primary users like $\mathrm{PU}=\left\{\mathrm{PU}_{1}\right.$, $\left.\mathrm{PU}_{2}, \ldots . . \mathrm{PU}_{\mathrm{i}}\right\}$. These are stationary users like our TV towers. In addition, we are having the $\mathrm{j}$ number of secondary users in both the licensed and unlicensed spectrum under the control of base stations. Whenever there is a need for end users, we can associate these to the groups for effective communication.

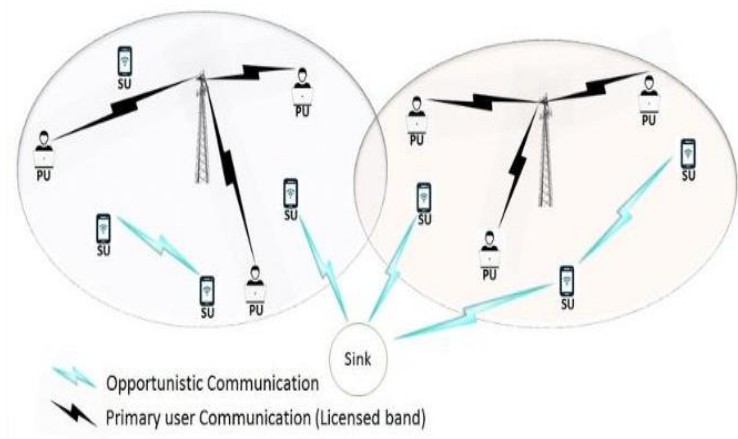

\section{Fig 1 :Structure of the proposed work}

The unlicensed users or SU's need to continuously monitor the spectrum for the presence of the licensed users (PU)with the help fo OFDM. If the PU is absent for a specific time, the SU can use that spectrum for transmission till the PU reappears. Once the PU reappears, the SU should handoff and yield that spectrum for the PU and will shift to another unused spectrum.This implies that the SU's should continuously monitor the entire spectrum for an opportunity to use a channel that is not being used by the PU.[1] The unused spectrums available in two cases, one is temporal unused spectrum, second one is spatial unused spectrum. A temporal unused spectrum appears when a PU does not transmit during a particular amount of time period and the SUs can use the spectrum for that time. A spatial unused spectrum appears

Copyright (C) Author when the PU transmits within a neighborhood and therefore the SUs can then use that spectrum outside that area. The spectrum sensing performance is affected by noise uncertainty, shadowing and multipath fading[9].

In spectrum sensing techniques we introduce the hypothesis test, based on which the performances of the techniques are tested. The hypothesis model is as follows:

$\mathrm{H} 0: \mathrm{y}(\mathrm{t})=\mathrm{n}(\mathrm{t})$,

$\mathrm{H} 1: \mathrm{y}(\mathrm{t})=\mathrm{h}^{*} \mathrm{x}(\mathrm{t})+\mathrm{n}(\mathrm{t})$

where $y(t)$ is that the received signal, $x(t)$ is that the primary user signal, $\mathrm{n}(\mathrm{t})$ is additive white Gaussian noise and $h$ is that the channel gain of PU.The hypothesis $\mathrm{HO}$ is a null hypothesis which means that there is no primary signal present whereas $\mathrm{H} 1$ indicates the presence of the primary signal.

\section{Sensing Based Cluster Formation}

In cooperative spectrum sensing, more than one SU and is committed with sensing and suggestion of the global result based on the SU of local reports. This leads to overhead in the fusion center which demean the network performance. As multiple SUs senses the same channel, cooperative sensing required more energy to perform[10]. To address these issues, we propose sensing based cluster formation; the whole network is organized several clusters on consideration of sensing ability of cognitive radio. Energy discernment is used for local sensing because of its simplicity and fast identification. At time Ts, cognitive radio senses the frequency range fi, since it receives the discrete time signal $\mathrm{X}(\mathrm{t})$ in this range. In traditional binary sensing, two levels of hypothesis testing $\left(\mathrm{H}_{0}, \mathrm{H}_{1}\right)$ derived from received signal are given as,

$$
\mathrm{x}(\mathrm{t})= \begin{cases}n(t), & H_{0} \\ h s(t)+n(t), & H_{1}\end{cases}
$$

Hypothesis $\mathrm{H}_{0}$ declares as noise only is present in that frequency rangef ${ }_{i}$. Noise 
occupation than with signal in $f_{i}$ is given as $\mathrm{H}_{1}$.

Our proposed concept is first select cluster head $(\mathrm{CH})$ on the basis of the high probability of both node's SINR $\rho$ n and a number of not active channel $\mathrm{C}$ at a specified range of sensing time Ts .Before they enter into cluster and their past history, every node has sensed and identified a different number of not active channelsCi .In Number of cluster heads define the number of cluster. Next elected $\mathrm{CH}$ broadcasts their request message (REQ) into its one-hope neighbor nodes. $\mathrm{CH}$ would construct a cluster that contains only nodes which replied (REP)to the request of $\mathrm{CH}$. Indirect communication between cluster member and fusion center obtained through cluster head less the chances of overhead at fusion center.

\section{Channel Allocation to SU}

Resource management controls the assignment of bandwidth and channel then in order to achieve spectral efficiency. Spectral efficiency is defined as the rate of information transmitted over given bandwidth and channel or save the spectrum in CRN.

We considered co-channel interference and adjacent channel interference when sharing with others. Control message of sensing reports and channel status information are to be transmitted over a common channel for all secondary nodes, also known as a control channel. In CRN, SU allowed to sense and utilize not active authorized spectrum under the control of the cognitive base station[11]. CBS had completely finished the status of the channel using local sensing reports. CBS will allocate a channel to SU, If channel status is free. To select worthy SU to stabilize the utilization of spectrum holes, CBS study three parameters: packet size, required bandwidth and node's SNR. The new system of CRN has a total M primary channel assigned to utilize $\mathrm{N}$ channels. These $\mathrm{N}$ channels are periodically sensed by $\mathrm{j}$ SUs and send a report to fusion center which decides the spectrum as not active or busy. Another responsibility of fusion center is to allocate a free channel to any one SU. The relation between packet size $(\mathrm{S})$, transmission rate $(\mathrm{R})$ and execution time (T) is given as,

$$
R=S / T
$$

SINR refers the ratio of RSS $(\mathrm{P})$ to interference (I) and noise $(\mathrm{N})$ at the receiver. Every node has a unique SINR that dependent on background noise and interference of another simultaneous communication is given as,

$$
\mathrm{SINR}, \rho=\mathrm{P} / 1+\mathrm{N}
$$

$B_{n}$ is the width of the frequency band that is enough to ensure the transmission of the grade required

Pseudo code for PRN channel allocation algorithm

Input: $\mathrm{A}_{\mathrm{n}}, \mathrm{SU}_{\mathrm{j}}$

Available channel $A_{n}=\left\{A_{1}, A_{2} \ldots \ldots \ldots . A_{n}\right\}$, $\mathrm{SU}_{\mathrm{j}}=\left\{\mathrm{SU}_{1}, \mathrm{SU}_{2} \ldots \ldots . . \mathrm{SU}_{\mathrm{j}}\right\}$

- For $\mathrm{SU}_{\mathrm{j}}$, compute $\mu$

- SorttheSU $\mathrm{J}_{\mathrm{j}}$ in ascending order based on the value of $\mu$

- Assign $\mathrm{A}_{\mathrm{n}}$ to $\mathrm{SU}_{\mathrm{j}}$

Estimate $\mathrm{R}$ for channel

- If $\mathrm{R}$ is desired,

$$
\begin{aligned}
& \mathrm{N}++; \\
& \mathrm{J}++; \\
& \text { Continue for assignment next }
\end{aligned}
$$

channel

- Otherwise

assignment

$$
\text { Only } \mathrm{N}++ \text {, go to channel }
$$

The data Packets are divided into three various sizes such as small, medium and 
bigger size packets. The usage of

Multichannel MAC protocol also results in efficient video transmission and so on.

\section{SIMULATION RESULTS:}

Results has be simulated using NS2 simulation software. The packet delivery ratio was figured out and it was shown in the figure below.

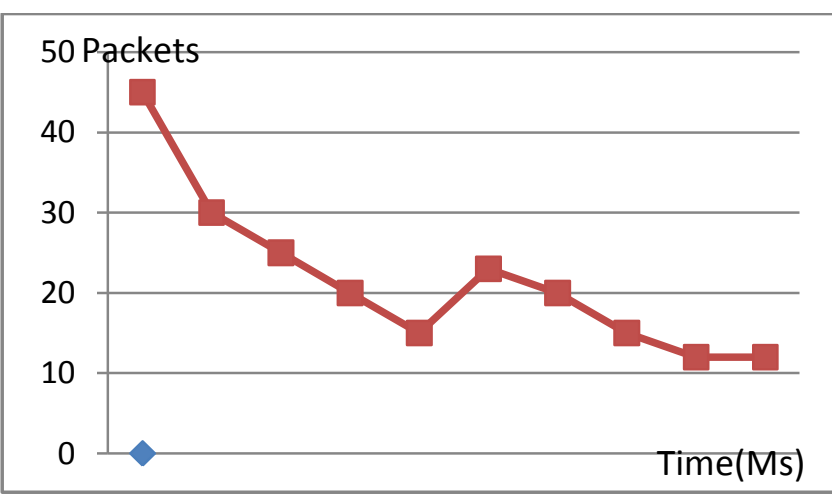

Fig2: Packet delivery ratio

By using medium size packets the data are transmitted efficiently with less time delay.The below figure shows the throughput of the data transmission.

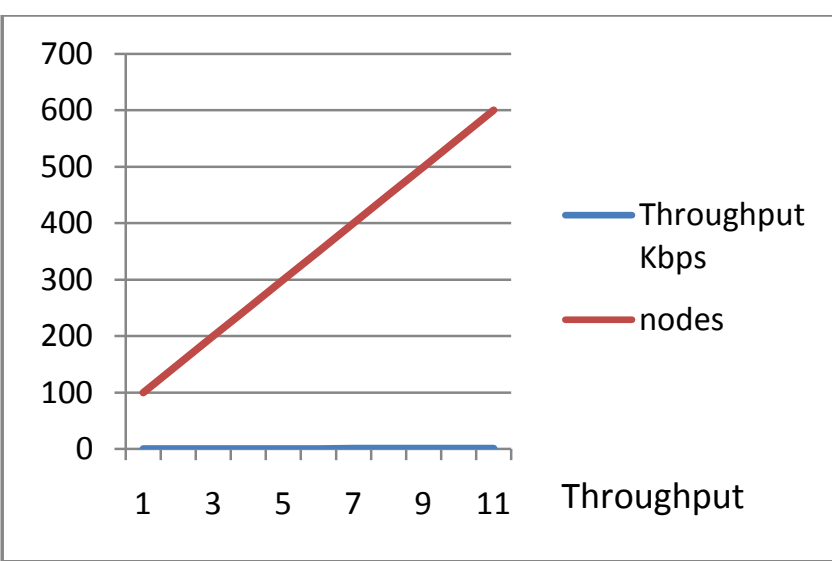

Fig 3: Throughput Vs Nodes

The above graph shows the increased performance of data packets. Throughput is increasing as the number of nodes increases. Thus the simulation results shows that the medium size packets are efficient in data transmission.

\section{CONCLUSION}

The size of the packet plays a vital role in video transmission and its output shows that the medium size packet gives more throughput and increase the channels utilization. Also our method improves output gain, less delay and energy efficiency. The Simulation results shows the performance metrics of medium size packets over other packets. Further the results prove that the large size packets do not enhance the throughput and efficiency

\section{REFERENCES:}

Mohammed

Ai-Medhwahi, FazirulhisyamHashim,

BorhanuddinMohd Ali and A.Sali "Impact of Packet size in Adaptive Cognitive Radio Sensor Network" Wireless communication and Mobile computing, volume 2018.

[2] Saleh Alrkiyan, WCDMA System, Department of Electrical Engineering, College of engineering, Qassim University, Kingdom of Saudi Arabia

[3] A.Arokkiaraj, T.Jaysankar, "OFDM Based Spectrum Sensing in Time Varying Channel", International Refereed Journal of Engineering and Science (IRJES), Volume 3, Issue 4(April 2014), pp.50-55,

[4] T. Jayasankar, M.Sujatha and C.Rajinikanth, "Unconstrained Global Optimization Base Partial Transmit Sequence For OFDM PAPR Reduction", International Journal of Advanced Science and Technology, Vol.29,No.3, (2020), pp.7346-7354

K. J. Kim, K. S. Kwak, and B. D. Choi, "Performance analysis of 
opportunistic spectrum access protocol for multi-channel cognitive radio networks," Journal of Communications and Networks, vol. 15 , no. 1, pp. 77-86, 2013.

[6] IEEE 802 Working Group, "Ieee standard for local and metropolitan area networks" part 15.4: Low-rate wireless per- sonal area networks (lrwpans)," IEEE Std, vol. 802, pp. 4 2011, 2011.

[7] P. Huang, L. Xiao, S. Soltani, M. W. Mutka, and N. Xi, "The evolution of MAC protocols in wireless sensor networks: a survey," IEEE Communications Surveys \& Tutorials, vol. 15, no. 1, pp. 101120, 2013.

[8] M. Sudha, J. Sundararaian“"ClusterBased Energy Hop Count Analysis for Dynamic Route Selection in Mobile Wireless Sensor Network" Applied Mathematics \& Information Sciences, Vol 13, no 4, pp. 611-619, 2019

[8] IEEE Standards Association, "Part 22: Cognitive wireless ran medium access control (mac) and physical layer (phy) specifi- cations: Policies and procedures for operation in the tv bands," IEEE Standard, vol. 802, 2011.

[9] D. Cabric, S. M. Mishra, and R. W. Brodersen, "Implementation issues in spectrum sensing for cognitive radios," in Proceedings of the Conference Record of the 38th Asilomar Conference on Signals, Systems and Computers, pp. 772776, Pacific Grove, Calif, USA, November 2004.

[10] W.-Y. Lee and I. F. Akyildiz, "Optimal spectrum sensing framework for cognitive radio networks," IEEE Transactions on Wireless Communications, vol. 7, no. 10, pp. 3845-3857, 2008.
[11] G. P. Joshi, S. Y. Nam, and S. W. Kim, "Cognitive radio wireless sensor networks: applications, challenges and research trends," Sensors, vol. 13, no. 9, pp. 11196-11228, 2013.

[12] J. Ren, Y. Zhang, N. Zhang, D. Zhang et al., "Dynamic channel access for energy efficient data gathering in cognitive radio sensor networks," https://arxiv.org/abs/1507.06188. 